\title{
The inner dust shell of Betelgeuse detected by polarimetric aperture-masking interferometry ${ }^{\star}$
}

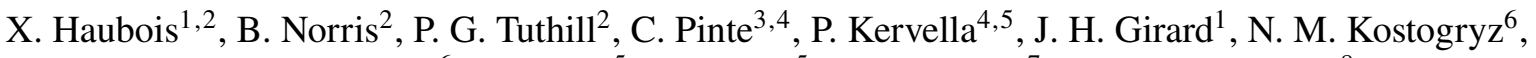 \\ S. V. Berdyugina ${ }^{6}$, G. Perrin ${ }^{5}$, S. Lacour ${ }^{5}$, A. Chiavassa ${ }^{7}$, and S. T. Ridgway ${ }^{8}$ \\ ${ }^{1}$ European Organisation for Astronomical Research in the Southern Hemisphere, Casilla 19001, Santiago 19, Chile \\ e-mail: xhaubois@eso.org \\ 2 Sydney Institute for Astronomy, School of Physics, University of Sydney, NSW 2006, Australia \\ ${ }^{3}$ UJF-Grenoble 1/CNRS-INSU, Institut de Planétologie et d'Astrophysique de Grenoble, UMR 5274, 38041 Grenoble, France \\ ${ }^{4}$ Unidad Mixta Internacional Franco-Chilena de Astronomía, CNRS/INSU UMI 3386 and Departamento de Astronomía, \\ Universidad de Chile, Casilla 36-D Santiago, Chile \\ ${ }^{5}$ LESIA, Observatoire de Paris, PSL Research University, CNRS, Sorbonne Universités, UPMC Université Paris 06, \\ Université Paris Diderot, Sorbonne Paris Cité, 5 place Jules Janssen, 92195 Meudon, France \\ ${ }^{6}$ Kiepenheuer-Institut für Sonnenphysik (KIS), Schöneckstrasse 6, 79104 Freiburg, Germany \\ ${ }^{7}$ Laboratoire Lagrange, Université Côte d'Azur, Observatoire de la Côte d'Azur, CNRS, Boulevard de l'Observatoire, CS 34229 , \\ 06304 Nice Cedex 4, France \\ ${ }^{8}$ National Optical Astronomy Observatory, PO Box 26732, Tucson, AZ 85726-6732, USA
}

Received 18 April 2018 / Accepted 30 June 2019

\begin{abstract}
Context. Theory surrounding the origin of the dust-laden winds from evolved stars remains mired in controversy. Characterizing the formation loci and the dust distribution within approximately the first stellar radius above the surface is crucial for understanding the physics that underlie the mass-loss phenomenon.

Aims. By exploiting interferometric polarimetry, we derive the fundamental parameters that govern the dust structure at the wind base of a red supergiant.

Methods. We present near-infrared aperture-masking observations of Betelgeuse in polarimetric mode obtained with the NACO/SAMPol instrument. We used both parametric models and radiative transfer simulations to predict polarimetric differential visibility data and compared them to SPHERE/ZIMPOL measurements.

Results. Using a thin dust shell model, we report the discovery of a dust halo that is located at only $0.5 R_{\star}$ above the photosphere (i.e. an inner radius of the dust halo of $1.5 R_{\star}$ ). By fitting the data under the assumption of Mie scattering, we estimate the grain size and density for various dust species. By extrapolating to the visible wavelengths using radiative transfer simulations, we compare our model with SPHERE/ZIMPOL data and find that models based on dust mixtures that are dominated by forsterite are most favored. Such a close dusty atmosphere has profound implications for the dust formation mechanisms around red supergiants.
\end{abstract}

Key words. techniques: interferometric - stars: fundamental parameters - infrared: stars - stars: individual: Betelgeuse

\section{Introduction}

Betelgeuse ( $\alpha$ Orionis) is a red supergiant (hereafter RSG) of spectral type M2Iab and one of the brightest stars in the night sky at all wavelengths. With a distance of $222_{-34}^{+48} \mathrm{pc}$ (Harper et al. 2017), its angular diameter of $\sim 43$ mas in the near-infrared (e.g., Perrin et al. 2004; Haubois et al. 2009; Montargès et al. 2014) makes it an ideal target for studying the inner structures that are involved in the poorly constrained mass-loss phenomenon. For an overview of Betelgeuse's properties, we refer to the proceedings of a workshop that focused on Betelgeuse (Kervella et al. 2013).

Betelgeuse exhibits a complex signal that is spectrally and temporally variable in net polarized light. From the temporal analysis of four years of $B$-band polarization data, the reported variability has been interpreted in terms of activity of large-scale

\footnotetext{
* Based on SAMPol data obtained at the ESO VLT Yepun telescope (090.D-0898(A)).
}

convective cells (Hayes 1984). UBV polarimetric measurements from $6^{\prime \prime}$ to $25^{\prime \prime}$ around the star (Le Borgne et al. 1986) led to the detection of a silicon dust environment whose grain size is in the range between 0.05 and $0.5 \mu \mathrm{m}$ (Mauron \& Le Borgne 1986).

Interferometric reconstructed images in the visible unveiled an elliptical structure around Betelgeuse at $\sim 2-2.5$ stellar radii (Roddier \& Roddier 1985). The favored explanation for this circumstellar emission was Mie scattering of stellar light by dust particles. This interpretation was found in agreement with Draine (1981), who showed that assuming a $3600 \mathrm{~K}$ effective temperature for Betelgeuse, clean silicate grains would start to condense at 1.8 stellar radius. This confirmed previous work predicting that at such a short distance above the photosphere, this dust shell would yield a significant degree of polarized light in the visible (Tsuji 1978). Based on interferometric and spectroscopic observations, Verhoelst et al. (2006) suggested that an amorphous alumina shell at $1.5 R_{\star}$ could account for the fact that Betelgeuse appears 1.5 times larger in the mid-infrared (MIR) than in the near-infrared (NIR). However, given the sublimation 
temperature of $1900 \mathrm{~K}$ for alumina, the gas pressure required to maintain such a shell is $10^{4}$ times higher than model predictions for the inner atmosphere of Betelgeuse. Perrin et al. (2007) found that a layer of water vapor coexists with a thin shell composed of $\mathrm{SiO}$ and alumina in a maximum radius of about 62.5 mas, corresponding to $1.43 R_{\star}$ in the $N$ band. Farther away at about arcsecond spatial scales, Kervella et al. (2011) reported MIR spectroimaging observations of the highly asymmetric Betelgeuse circumstellar environment, concluding that silicates or alumina dust were viable constituents.

The atmosphere of Betelgeuse is subject to $\sim 1 \mathrm{G}$ magnetic fields inferred from the Zeeman effect (Aurière et al. 2010), which are thought to originate from local low-scale convective activity. Simplifying the interpretation of polarized-light signals, such modest fields are unlikely to result in significant levels of direct continuum polarization. Extreme adaptiveoptics polarimetric observations within the Spectro-Polarimetric High-contrast Exoplanet Research (SPHERE) obtained with the Zürich Imaging Polarimeter (ZIMPOL) were obtained by Kervella et al. (2016), resolving the surface and inner environment of Betelgeuse. A somewhat complex picture emerged from this work, in which media of inhomogeneous densities and temperatures coexist in asymmetric structures. Dust is confirmed at $3 R_{\star}$, but the authors note that polarization levels might be diluted by (unpolarized) gaseous emission, which allows for the possibility that significant dust may exist interior to this.

Developments in polarimetric aperture masking interferometry (SAMPol mode at NACO) allowed novel polarized-light studies of dust shells around AGB stars (Norris et al. 2012). Observational studies targeting several bright systems revealed dust shells capable of strong scattered-light interaction with the stellar radiation field. These systems had typical diameters of about $2 R_{\star}$ and grain sizes of about $300 \mathrm{~nm}$. These data immediately provoked renewed interest in the role played by photon scattering in the physics of radiatively driven winds, opening a new window to explore links between the stellar properties and final mass-loss rates on the AGB.

Based on the same observing technique, we aim to identify the nature and the location of the polarizing structures at the wind base for the red supergiant Betelgeuse. We here report on optical interferometry polarimetric observations of Betelgeuse obtained with the NACO/SAMPol instrument. Section 2 describes the observations and the data reduction. In Sect. 3 we present the detection of a thin polarizing dust shell. In Sect. 4 radiative transfer modeling is used to reproduce the SAMPol observations and compare them to the visible observations made with ZIMPOL. In Sect. 5 we discuss the effects of polarization that stem from the stellar atmosphere before we conclude in Sect. 6.

\section{Observation and data reduction}

Betelgeuse was observed with NACO/SAMPol on 28 January 2013. The instrument was configured to perform aperturemasking interferometry (SAM mode; Lenzen et al. 2003; Rousset et al. 2003) together with the use of a Wollaston prism and rotating half-wave plates (HWP), which together comprise the supported "SAMPol mode". Following the work of Norris et al. (2012), the extreme spatial resolution delivered by SAM in concert with differential polarimetric data is capable of revealing scattered-light structures at spatial scales that are not accessible to competing technologies. Despite strongly polarized individual constituent components, an astrophysical image will often yield zero net polarization as a sum over a circularly symmetric environment. Operating at the extreme spatial resolution limit, SAMPol is capable of breaking the degeneracy, delivering information on polarized structures at spatial scales that are normally hidden.

This work employed the 18-hole aperture mask (net transmission of $3.9 \%$ with respect to the full aperture), with an integration time of $0.1 \mathrm{~s}$ obtained with a subframe window of $512 \times 514$ pixels on the Aladdin 3 detector. For each image data cube, interferograms for the two orthogonal polarizations split by the Wollaston prism are recorded simultaneously on the detector array. The full starlight polarization state is explored by changing the rotation angle of upstream half-wave plates. This experiment employed four half-wave plate positions separated by $22.5^{\circ}$ in order to sample the $Q$ and $U$ Stokes parameters. Following a relatively standard data reduction procedure entailing sky subtraction, flat fielding, and cosmic-ray removal, the complex visibilities and bispectra were separately accumulated for each baseline and baseline triplet, respectively, for each of the two polarizations. Finally, in order to remove systematics that are due to the non-common paths after the Wollaston prism, horizontally polarized visibilities $\left(V_{\mathrm{h}}\right)$ were divided by vertically polarized visibilities $\left(V_{\mathrm{v}}\right)$, forming a single differential observable. This ratio of the observables, hereafter called differential (polarimetric) visibility, is highly robust to errors induced by seeing because the two polarizations were recorded simultaneously. A list of spectral filters employed for the SAMPol observations is given in Table 1 .

\section{Detection of the dusty inner environment of Betelgeuse}

Although strictly speaking, the self-calibrating differential polarimetric visibility obviates the need for the usual strict observance of known (usually point source) stars for the system transfer function, here the dust-free red giant Aldebaran was chosen as a non-polarized reference object. Photometric measurements performed in the optical and infrared domains (Ducati 2002) were compared with a simple (non-dusty) synthetic spectrum obtained from Castelli \& Kurucz (2003) ${ }^{1}$ and with the following parameters: effective temperature $T_{\text {eff }}=3900 \mathrm{~K}$, $\log g=1.5$ and limb-darkened disk with a diameter of 20.60 mas (Fig. 1). The $\chi^{2}$ per data point (equivalent of a reduced $\chi^{2}$ ) of the comparison between the Kurucz spectrum and the photometric measurements is equal to 1.95 , meaning that no significant infrared excess is present in this part of Aldebaran's spectrum. Furthermore, no infrared excess was detected between 8 and $13 \mu \mathrm{m}$ (Monnier et al. 1998), nor between 50 and $670 \mu \mathrm{m}$ (Dehaes et al. 2011).

Differential polarized visibility $\left(V_{\mathrm{h}} / V_{\mathrm{v}}\right)$ curves for Betelgeuse (left panel) and Aldebaran (right panel) are presented in Fig. 2 for the filter centered on $1.04 \mu \mathrm{m}$. The corresponding curves for other filters are presented in Figs. A.1 and A.2. Any significant departure from the value of unity over a range of baselines indicates that a polarized structure has been resolved. The varying signal apparent for Betelgeuse (but not Aldebaran) can be first approximated by a sinusoid, and is the first-order expectation for any spherically symmetric structure (see also Norris et al. 2012). We note that the amplitude of this sinusoidal variation decreases with observing wavelength: the filters with central wavelength above $1.75 \mu \mathrm{m}$ show no sign of a polarized signal.

\footnotetext{
1 Accessible from http://www.stsci.edu/hst/observatory/ crds/
} 
Table 1. Characteristics of spectral filters employed for the SAMPol observations.

\begin{tabular}{lccc}
\hline \hline Filter & Central wavelength $(\mu \mathrm{m})$ & Width $(\mu \mathrm{m})$ & Observed source $(\mathrm{s})$ \\
\hline NB 1.04 & 1.040 & 0.015 & Betelgeuse/Aldebaran \\
NB 1.08 & 1.083 & 0.015 & Betelgeuse \\
NB 1.09 & 1.094 & 0.015 & Betelgeuse/Aldebaran \\
NB 1.24 & 1.237 & 0.015 & Betelgeuse/Aldebaran \\
NB 1.28 & 1.282 & 0.014 & Betelgeuse \\
NB 1.64 & 1.644 & 0.018 & Betelgeuse/Aldebaran \\
NB 1.75 & 1.748 & 0.026 & Betelgeuse/Aldebaran \\
NB 2.12 & 2.122 & 0.022 & Betelgeuse/Aldebaran \\
IB 2.30 & 2.30 & 0.06 & Betelgeuse/Aldebaran \\
IB 2.36 & 2.36 & 0.06 & Betelgeuse/Aldebaran \\
IB 2.42 & 2.42 & 0.06 & Betelgeuse/Aldebaran \\
\hline
\end{tabular}

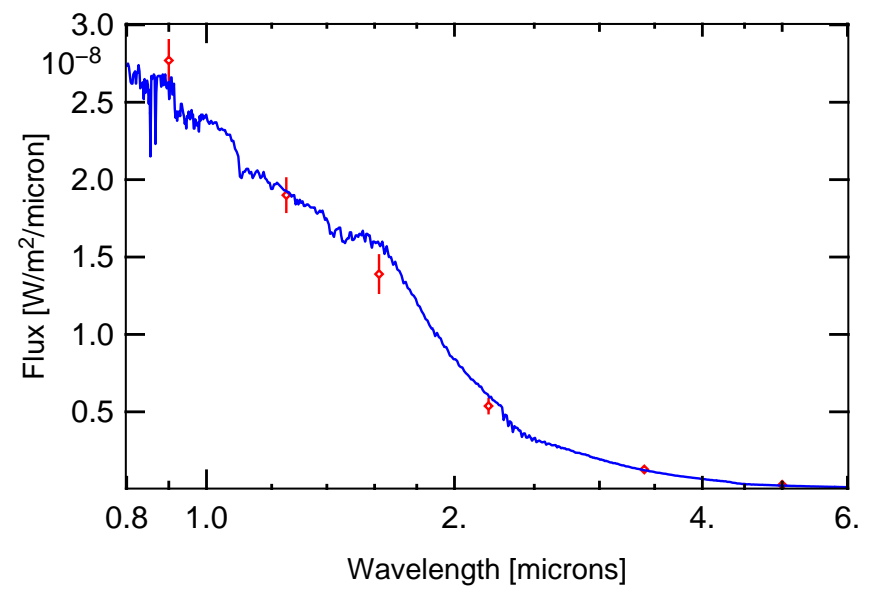

Fig. 1. Spectral energy distribution of Aldebaran. Red points correspond to observed magnitudes reported in Ducati (2002) The blue line shows a synthetic model with $T_{\text {eff }}=3900 \mathrm{~K}, \log g=1.5$, and a limb-darkened disk diameter of 20.60 mas.

This is expected from signals with an astrophysical origin in which the dust grain-size distribution enforces a strong spectral dependence of the polarization. Although this provides a strong affirmation that the SAMPol data have reach into the physics of the circumstellar dust halo, it also means that the longer wavelength data with null signals have scant ability to constrain the models, and are therefore largely discarded in the subsequent analysis.

It is also worth emphasizing that compared to Betelgeuse, Aldebaran shows no significant variation of differential visibility, neither as a function of baseline length nor of azimuth angle. The finding of a null result for polarimetric signals that are detected by observing a non-dusty source lends enormous confidence that no major instrumental polarization biases or uncalibrated systematic errors remain to contaminate the data when we consider our results on Betelgeuse.

\subsection{Dust shell radius}

The amplitude of the differential polarized visibility signal is about $4 \%$ at $1.04 \mu \mathrm{m}$ for Betelgeuse, in accord with theoretical predictions from NIR scattered-light models on a thin dust shell (Sect. 3.3 of Haubois et al. 2009). Because the dominant term is a sinusoidal variation of the differential polarized visibility curves with azimuth (Figs. 2, A.1, and A.2), the simplest first-order model to produce such a polarized signal is a spherical dust shell that scatters the stellar light. This model entails only three parameters: the stellar uniform-disk radius $R_{\star}$, the dust shell radius $R_{\text {dust }}$, and the flux ratio between the two structures. Hereafter we term the latter the "scattered-light fraction" because it represents a proxy for the ratio between the flux radiated from gas and the scattered flux from dust. It is important to emphasize that we define this fraction for the polarized light alone. In this model, the shell thickness is negligible compared to its diameter. The model computes differential polarized visibilities from images that are simulated in the two orthogonal polarizations, allowing fits to the observed primary observables. This model was previously used in Ireland et al. (2005) and in Norris et al. (2012). Because simple circular symmetry is assumed, differential polarized visibilities could easily be reduced to a single azimuthally symmetric quantity, permitting the model to be parameterized as a function of baseline length alone. An example best-fit model is presented in Fig. 3, and the corresponding 2D image from this parametric model is shown in Fig. 4. Model results for all filters are summarized in Table 2.

The model describing the underlying stellar brightness distribution was found to have very little impact on the fits. Stellar models employing a uniform disk gave identical results to those assuming a limb-darkened disk. This can be explained by the relatively limited angular resolution intrinsic to the SAMPol data: no second and higher lobes of the visibility function are probed, where the detailed form of the brightness profile is important.

The uncertainty in the fit parameters increases with wavelength as the polarized signal decreases. Taking into account the three short-wavelength spectral filters, we find that $R_{\text {dust }} \approx$ $1.5 R_{\star}$. The finding of strong scattered-light signals from dust in such proximity to the stellar surface constrains the dust chemistry. In the following, we consider three types of dust species found in O-rich evolved stars, all of which have high sublimation temperatures: $\mathrm{MgSiO}_{3}$ (enstatite), $\mathrm{Mg}_{2} \mathrm{SiO}_{4}$ (forsterite), and $\mathrm{Al}_{2} \mathrm{O}_{3}$ (alumina).

\subsection{Modeling the scattered-light fraction}

As previously presented in Norris et al. (2012), the decrease in the scattered-light signal with increasing wavelength can be reproduced by a Mie scattering opacity. Denoting photons scattered by dust as $I_{\text {dust }}$, we employ the same model, where the scattered-light fraction can be written as

$\frac{I_{\text {dust }}}{I_{\text {total }}}=\frac{B\left(1-\mathrm{e}^{-\tau_{\mathrm{sc}}}\right)}{\mathrm{e}^{-\tau_{\mathrm{sc}}}+B\left(1-\mathrm{e}^{-\tau_{\mathrm{sc}}}\right)}$, 

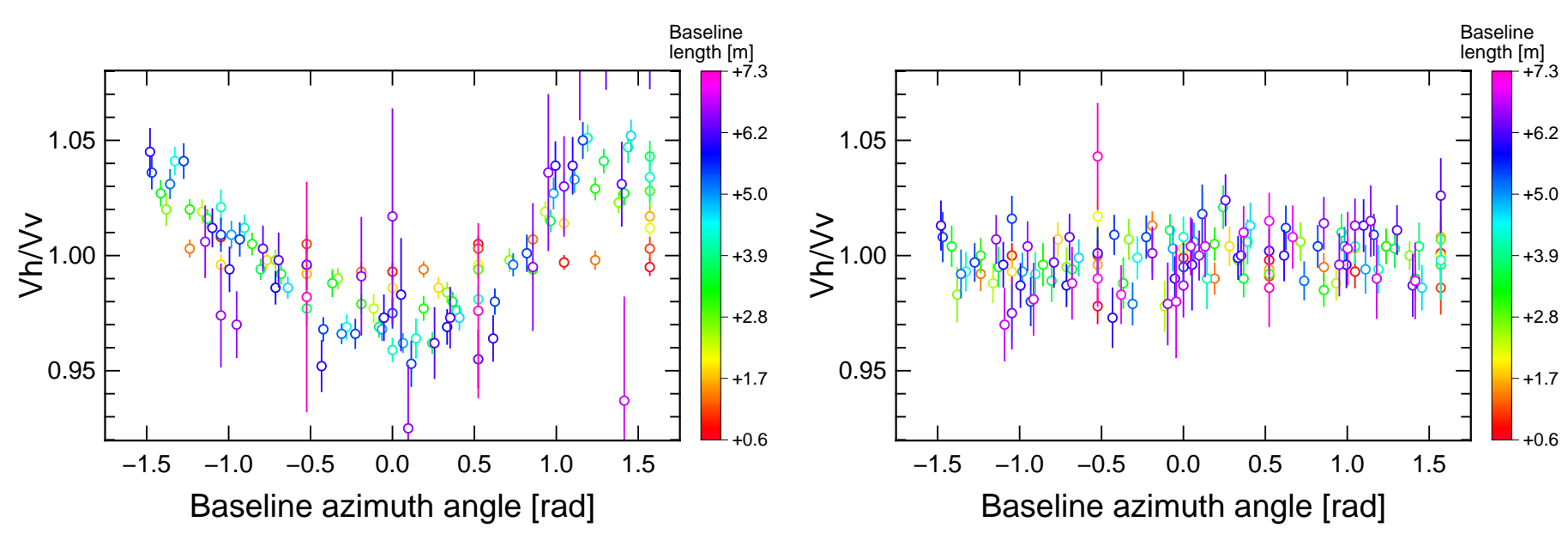

Fig. 2. Differential visibility ratio plotted as a function of azimuth angle, color-coded with baseline length for the filter centered on $1.04 \mu \mathrm{m}$. Left: Betelgeuse. Right: Aldebaran.

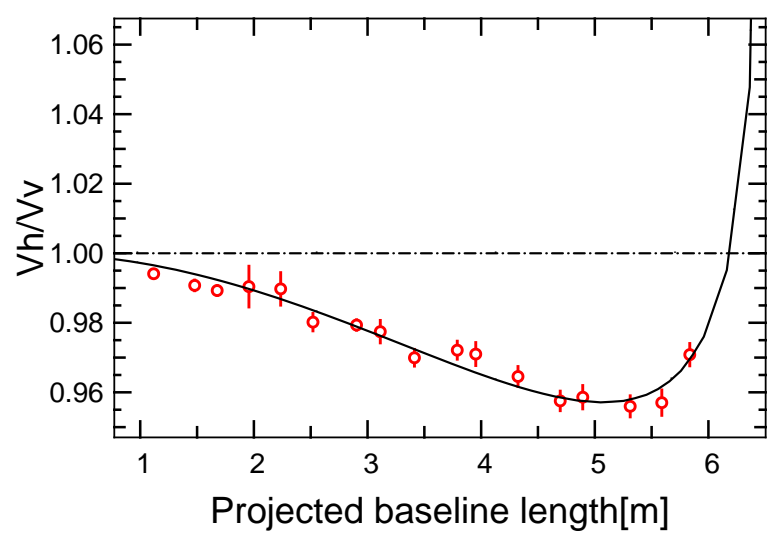

Fig. 3. Azimuthally reduced differential visibilities as a function of projected baseline length at $1.09 \mu \mathrm{m}$. SAMPol data are shown in red, and the dust shell model is plotted as a black line.

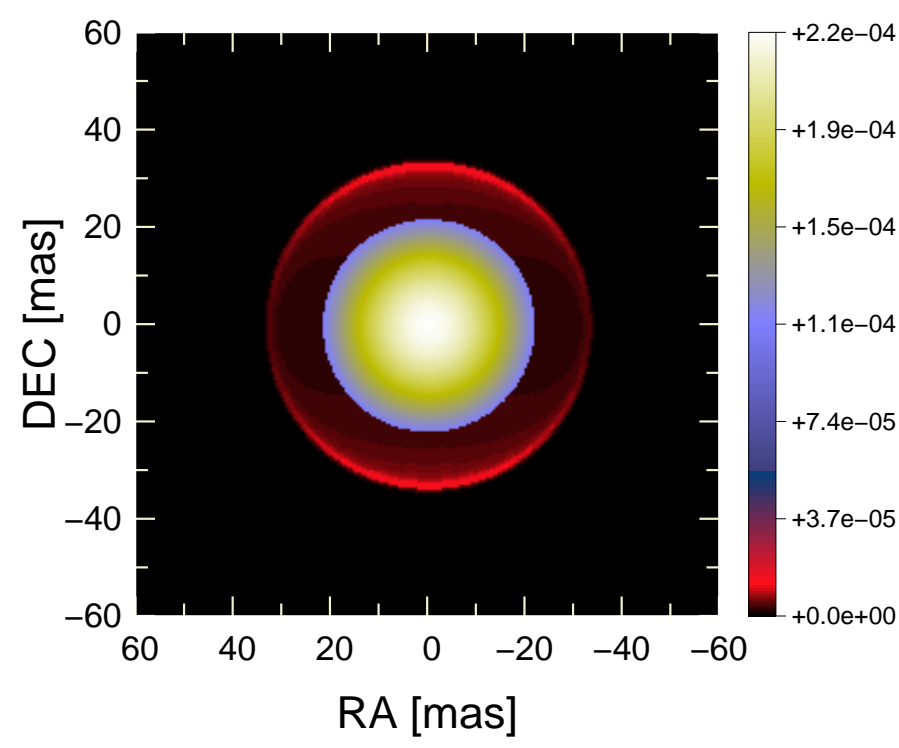

Fig. 4. Image of the dust shell model in the horizontal polarization at $1.09 \mu \mathrm{m}$. We used the parameters that correspond to the best fit as reported in Table 2. The intensities are normalized to the total intensity of the image. where $\tau_{\mathrm{sc}}$ is the product of the Mie scattering cross section (computed using numerical routines made available by the Earth Observation Data group from the University of Oxford ${ }^{2}$ ) and of the surface grain density. The term $B$ takes into account the fraction of light that is intercepted by the star after it is back-scattered from the dust shell. The optical constants of the enstatite and forsterite species were derived from Jäger et al. (2003) and taken from the Jena University Database ${ }^{3}$. For $\mathrm{Al}_{2} \mathrm{O}_{3}$, we used the optical constants distributed in the MCFOST (Pinte et al. 2009) and ProDiMo (Woitke et al. 2009) modeling codes.

The outcome of this modeling, as shown in Fig. 5, is that the SAMPol measurements are well reproduced by the thin dust shell model. Table 3 presents the values of the best-fit parameters.

The size of the dust grains and the mass of the shell are similar to those for the AGB stars reported in Norris et al. (2012). For oxygen-rich AGB stars, one scenario proposes that photon scattering by Fe-free silicate grains can couple the momentum of the radiation field to the dust. This mechanism is able to launch a dusty wind provided the grain size is about $1 \mu \mathrm{m}$ (Höfner 2008). Based on the grain size estimation derived from our data, this scenario seems to be plausible for Betelgeuse as well and might even hold for other oxygen-rich red supergiants. However, the composition of such grains remains to be determined, and the question of how they can survive in such proximity to the stellar surface needs to be addressed.

Kervella et al. (2016) published observations of Betelgeuse with the SPHERE/ZIMPOL instrument, finding an asymmetric envelope where the degree of linear polarization peaks at a radius of about three times the $K$-band limb-darkened radius, that is, $\approx 65$ mas (see Fig. 7 of that paper). Assuming the NIR dust shell that we characterized with SAMPol data is part of the same envelope seen in the visible, we can extrapolate the properties of our three dust models to visible wavelengths and compare them to the ZIMPOL measurements.

\section{Comparison with visible polarimetric measurements}

In order to construct a framework in which polarization models could be compared with both SAMPol and ZIMPOL data,

\footnotetext{
2 http://eodg.atm.ox.ac.uk/MIE/index.html

3 http://www.astro.uni-jena.de/Laboratory/OCDB/

amsilicates.html
} 
Table 2. Model parameters fit to the differential visibilities in the filters where a polarized signal was detected.

\begin{tabular}{lcccc}
\hline \hline Filter & $R_{\star}($ mas $)$ & $R_{\text {dust }}($ mas $)$ & Scattered-light fraction $(\%)$ & $\chi_{\text {reduced }}^{2}$ \\
\hline NB 1.04 & $22.00 \pm 0.24$ & $32.35 \pm 0.17$ & $4.4 \pm 0.3$ & 3.2 \\
NB 1.08 & $22.31 \pm 0.23$ & $34.53 \pm 0.12$ & $4.2 \pm 0.1$ & 0.5 \\
NB 1.09 & $22.31 \pm 0.23$ & $33.22 \pm 0.10$ & $4.1 \pm 0.1$ & 1.8 \\
NB 1.24 & $23.84 \pm 0.34$ & $29.20 \pm 5.22$ & $2.2 \pm 0.8$ & 4.9 \\
NB 1.64 & $22.36 \pm 0.80$ & $27.41 \pm 4.02$ & $2.0 \pm 0.8$ & 2.8 \\
NB 1.75 & $23.30 \pm 0.90$ & $29.62 \pm 5.24$ & $1.5 \pm 0.5$ & 2.2 \\
\hline
\end{tabular}

Table 3. Results of fitting the Mie scattering shell model with different dust species.

\begin{tabular}{lcccc}
\hline \hline Dust species & Grain radius $(\mathrm{nm})$ & Surface grain density $\left(10^{6} \mathrm{~cm}^{-2}\right)$ & Dust shell mass $\left(10^{-10} M_{\odot}\right)$ & $\chi_{\text {reduced }}^{2}$ \\
\hline Alumina & $285.7 \pm 13.2$ & $6.1 \pm 1.1$ & $1.49 \pm 0.13$ & 1.1 \\
Forsterite & $320.0 \pm 20.3$ & $6.5 \pm 1.6$ & $1.58 \pm 0.19$ & 0.8 \\
Enstatite & $335.9 \pm 24.5$ & $6.8 \pm 1.9$ & $1.64 \pm 0.23$ & 0.7 \\
\hline
\end{tabular}

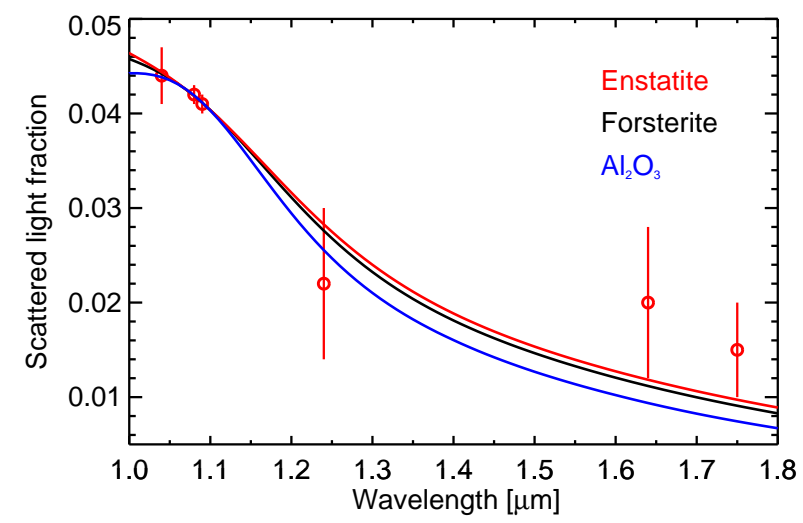

Fig. 5. Scattered-light fraction measured by SAMPol (red diamonds) with best-fit thin shell models based on the Mie scattering model for three dust species. The red error bars correspond to the $1 \sigma$ uncertainty of the measurements.

the radiative transfer code MCFOST (Pinte et al. 2006, 2009) was employed. By constructing a set of wavelengths spanning a given filter bandpass, images were accumulated using weights given by the spectral transmission profile of the filter. From the simulated images, model predictions could be derived for direct comparison with ZIMPOL and SAMPol observables.

As a sanity check, we ensured that we were able to reproduce the parametric modeling of the scattered-light fraction presented above. This is demonstrated in Fig. 6, where the curves were produced using model parameters found in Table 4 and assuming Mie scattering in a thin ( 0.05 au wide) dust shell. The only free parameter was the dust shell mass, which we tuned to match the parametric modeling curves (Fig. 5). For dust composed of forsterite and alumina, these values were within the $1 \sigma$ uncertainty found on the dust shell mass with the parametric modeling (Table 3), and marginally worse for the enstatite. These new values for the dust shell mass are presented in Table 4.

Employing the same parameters we used previously to produce the Mie scattering parametric models, we computed the degree of linear polarization as described in Kervella et al. (2016) in the four spectral filters. It is important to note that the maximum levels of the ZIMPOL degree of linear polarization are found at a radial distance between 60 and 80 mas. Therefore,

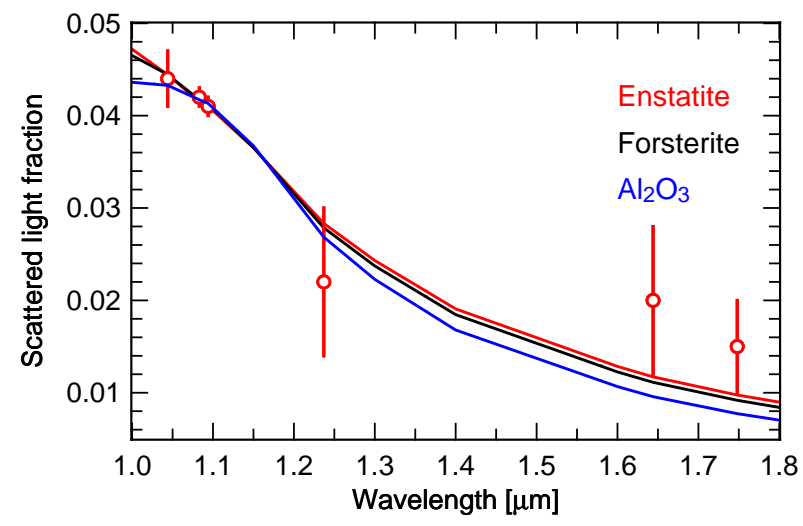

Fig. 6. Same as Fig. 5, but the curves for the scattered-light fraction were computed with the radiative transfer code MCFOST using the parameters listed in Table 4 and a Mie scattering. This accurately reproduces the parametric curves presented in Fig. 5.

we cannot directly compare the absolute values with those predicted by our dust models, where the shell is located at around 33 mas. Absolute values for the degree of linear polarization are indeed very sensitive to the grain density, the dust shell radius, and thickness. However, we can compare the variation in functional form with wavelength because this is sensitive to the dust composition. From this plot, given in Fig. 7, it is apparent that the variation in the ZIMPOL maximum degree of linear polarization is better reproduced across the red spectral region by enstatite or forsterite, but the $V$-band data point is better reproduced by the alumina.

MCFOST computes the dust temperature using the Monte Carlo method. At 7.3 au, the dust grain temperatures are $1650 \mathrm{~K}$, $1250 \mathrm{~K}$, and $750 \mathrm{~K}$ for the enstatite, forsterite, and alumina species, respectively. For enstatite, the temperature seems too high by a few hundred Kelvin to allow for dust condensation (Jäger et al. 2003), even if the required constituent atoms were available in sufficient numbers and for a time that is long enough for grain growth. Taken at face value, this would therefore rule out an in situ formation for this dust shell located at $1.5 R_{\star}$. The case is less clear for forsterite, but on the other hand, the condensation of alumina would seem definitely plausible based on the temperature value alone. 
Table 4. Parameters describing the star and dust shell models used for MCFOST simulations.

\begin{tabular}{lc}
\hline \hline MCFOST parameters & Values \\
\hline Distance & $222 \mathrm{pc}$ \\
Effective temperature & $3600 \mathrm{~K}$ \\
Stellar radius & $900 R_{\odot}$ \\
Stellar mass & $15 M_{\odot}$ \\
\hline Dust shell radius & $7.3 \mathrm{au}$ \\
Dust shell mass & $1.38 \mathrm{e}-10 M_{\odot}$ (alumina); $2.02 \mathrm{e}-10 M_{\odot}$ (enstatite); $1.72 \mathrm{e}-10 M_{\odot}$ (forsterite) \\
Minimum grain size & $0.284 \mu \mathrm{m}$ (alumina); $0.324 \mu \mathrm{m}$ (enstatite); $0.310 \mu \mathrm{m}$ (forsterite) \\
Maximum grain size & $0.298 \mu \mathrm{m}$ (alumina); $0.348 \mu \mathrm{m}$ (enstatite); $0.330 \mu \mathrm{m}$ (forsterite) \\
\hline
\end{tabular}

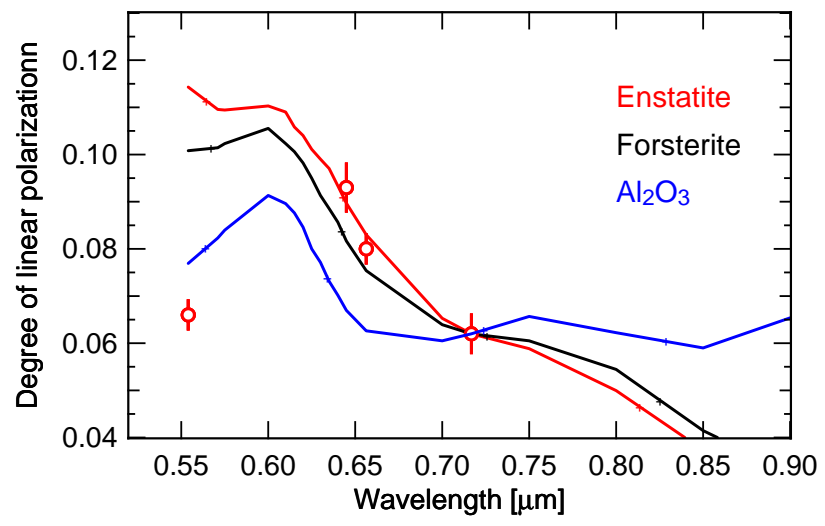

Fig. 7. Degree of linear polarization as a function of wavelength. Red circles indicate the maximum ZIMPOL data points reported in Fig. 7 of Kervella et al. (2016). With the goal of comparing their relative variation, the three dust models were offset with a constant so that their $0.717 \mu \mathrm{m}$ value matches the ZIMPOL datapoint $(0.0012,0.0077$, and 0.0231 for enstatite, forsterite, and alumina, respectively).

Given its extreme simplicity, the model shows promise that better matches might be obtained with the addition of one or a combination of further factors. We list these factors below.

- There may be a dust species or a combination of dust species that we did not consider here and that better reproduces the ZIMPOL measurements.

- Some effects that we did not model with MCFOST (nonspherical grains, hollow spherical grains, etc) could alter the outcomes.

- The $V$-band filter has a larger spectral bandwidth $(80 \mathrm{~nm})$ than the other filters. Several chromatic effects, both instrumental and/or astrophysical, may be at play to generate a lower degree of polarization for this datum.

- The structure imaged by ZIMPOL may not have the same composition and characteristics as the dust seen in the NIR shell. The ZIMPOL polarized images show that the dust atmosphere is clearly extended and deviates from the thin dust layer that we used to compare with the NIR measurements.

\section{Discussion}

\subsection{Polarization from the stellar atmosphere}

The stellar atmosphere is another potential source of polarization. It is therefore important to investigate its effects on the observables we analyzed previously.
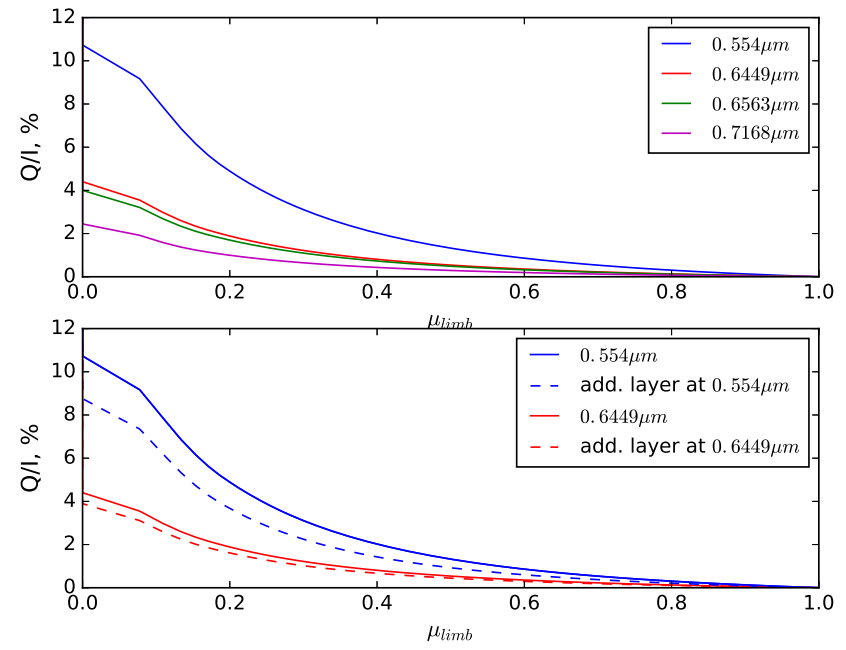

Fig. 8. Limb-darkening polarization, intensity-normalized Stokes $Q$ parameter as a function of $\mu_{\text {limb }}$. Upper panel: these curves are presented in four filters for the stellar atmosphere only. Lower panel: additional molecular layer has been added to the stellar atmosphere for two of the filters.

Center-to-limb polarization variations (CLPVs) of stellar atmosphere calculations in $\mathrm{F}, \mathrm{G}$, and $\mathrm{K}$ type stars were presented in Kostogryz et al. (2016). We adapted these calculations to the case of Betelgeuse and used the following stellar parameters: $M=15 M_{\odot}, R=1000 R_{\odot}, T_{\text {eff }}=3600 \mathrm{~K}$, and $\log g=0.0$.

The upper panel of Fig. 8 shows the limb-darkening fractional polarization intrinsic to the stellar atmosphere as a function of $\mu_{\text {limb }}$, which corresponds to $\mu(\mu=\cos (\theta)$, where $\theta$ is the angle between the line-of-sight direction and the direction normal to the stellar atmosphere), except that it ranges from where the largest gradient of the center-to-limb variation of intensity is observed (definition of the position of the observed stellar limb, see Sect. 3.1.2 of Kostogryz et al. 2016). The position of the observed stellar limb then corresponds to $\mu_{\text {limb }}=0$ and the normal to the atmosphere corresponds to $\mu_{\text {limb }}=1$. This plot shows that the limb-darkening fractional polarization has a declining strength to longer wavelengths (plots are tailored for the specific ZIMPOL filters we employed).

The lower panel reveals the effect on this quantity of a molecular layer (optical thickness of 0.01 , distance of 1.5 stellar radius) in addition to the stellar atmosphere for two selected filters: multiple scattering causes the fractional polarization to decrease up to a few percent depending on the wavelength.

We next integrated these CLPVs into our MCFOST models to take both the stellar atmosphere and the dust shell in the net 


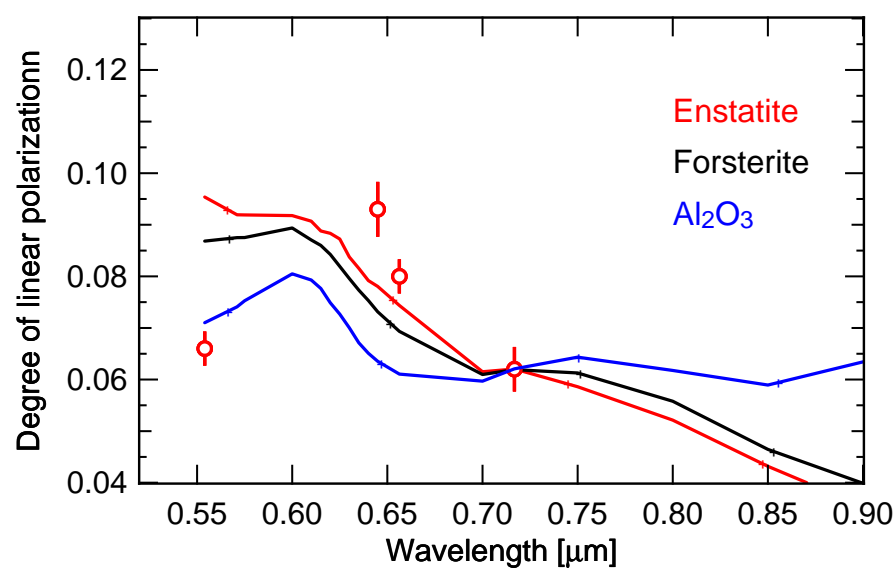

Fig. 9. Same as Fig. 7, with the addition of polarization stemming from the stellar atmosphere.

polarization computation into account. Figure 9 shows the same quantity as Fig. 7, but the CLPV is now included in the calculation of the Stokes parameters.

The main consequence of the addition of the CLPVs into our models is a global decrease in the degree of linear polarization at the visible wavelengths. As demonstrated in the lower panel of Fig. 8 with a molecular layer, this decrease can be attributed to the multiple scattering that takes place in a layer that is added to the stellar atmosphere. All models now clearly underestimate the observation data points at 0.6449 and $0.65634 \mu \mathrm{m}$. However, with this global decrease, the alumina model is able to better reproduce the $0.554 \mu \mathrm{m}$ data point. Several explanations for this inclusion of the CLPVs can be invoked to explain the overall poorer modeling of the ZIMPOL degree of linear polarization.

- The degree of linear polarization observed with ZIMPOL contains a very weak direct polarization signal from the stellar atmosphere because the wider circumstellar dust is well separated from the star at the instrumental resolution.

- The models with the lowest value of $\log g$ have $\log g=0.0$, while Betelgeuse has $\log g$ in the $[-0.2:-0.5]$ range.

- CLPV calculations were made for a homogeneous atmosphere, which (at some level) is not the case for Betelgeuse. A modeling effort taking the temperature distribution predicted by hydro-radiative simulations into account is ongoing.

We conclude that the additional complexity of adding stellar photosphere CLPVs to the modeling has done little to improve the ability to reproduce the wavelength dependence that is evident in the ZIMPOL data.

\subsection{Shell extension and mass-loss rates}

The dust shell masses reported here are lower than the massloss rates derived from infrared excesses (e.g., van Loon et al. 2005). However, interferometric polarimetry is most sensitive to thin dusty structures in close proximity to the star because they will provide the strongest polarized signal. The thin dust shell model invoked here might even represent a dense inner rim of a more extended envelope whose full extent is not easily revealed with this technique. This would explain why we see a thin dust shell and not a continuous structure as found by ZIMPOL, and also why we derive a relatively low mass-loss rate. We may only witness a part of the dust population located at the inner base of a larger envelope.

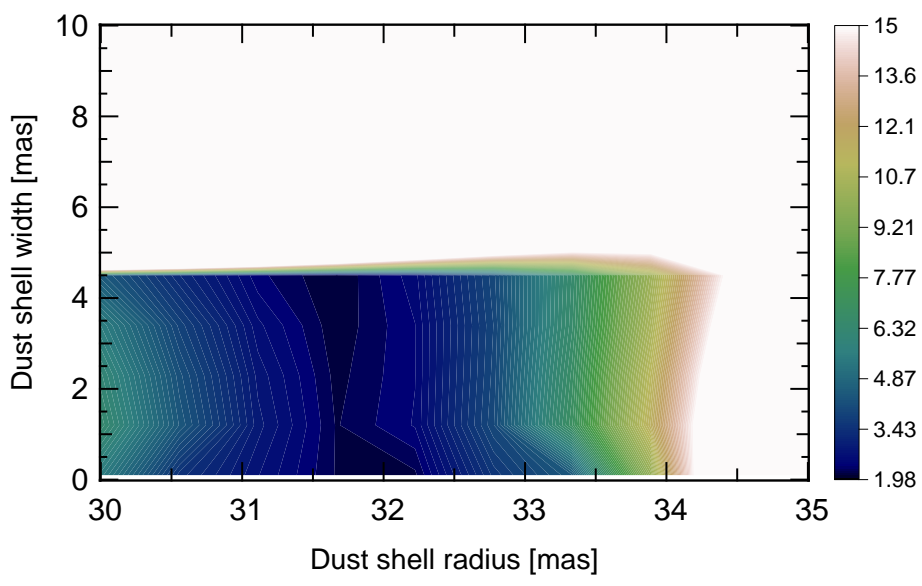

Fig. 10. Map of reduced $\chi$-squared for an extended dust shell model at $1.09 \mu \mathrm{m}$.

We attempted to model the $\mathrm{Vh} / \mathrm{Vv}$ data (see Sect. 3.1) using an extended shell model with a Gaussian density distribution. The reduced $\chi$-squared map is presented in Fig. 10. The best $\chi$-squared values we achieved are higher than for the thin shell model, even though we added the thickness (Gaussian FWHM) parameter. We note a large drop in $\chi$-squared values above a shell thickness of $\sim 4.5$ mas $(\sim 1$ au at $222 \mathrm{pc}$ ). For this extended model, the best fit to the data is obtained for apparent dust shell radii lower than the values reported in Table 2. We find that an apparent dust shell radius of 32 mas and a shell thickness of 4.5 mas with an FWHM lead to a density decrease of $\sim 10 \%$ in order to reproduce SAMPOL data, whereas the size of the dust grain has to be increased by $20 \%$ at most.

\subsection{Comparison with AGBs}

The many similarities of the astrophysical environments make a comparison between AGBs and RSGs very relevant in interpreting the properties of dust found close to the stellar surface. Recent studies, particularly using visible and NIR polarimetry, have shown that dust with grain sizes of a few tenths of a micron is common below $2 R_{\star}$; this conclusion seems to hold for varying mass-loss rates (Norris et al. 2012; Scicluna et al. 2015; Ohnaka et al. 2016; Khouri et al. 2016). We here add similar findings for the case of Betelgeuse. A common mechanism to explain dust nucleation and formation, despite differences in temperature, chemical composition, and stellar dynamics, might be indicated. Multi-epoch campaigns now offer the opportunity to link grain size variations with the AGB pulsation cycle (Ohnaka et al. 2017). However, in the case of RSGs, no strong pulsation analogous to that in AGBs is present, and time variability is rather dominated by convection time scales. A further extension of our study would therefore be to quantify the time variability of dust characteristics in the first stellar radii of Betelgeuse.

\section{Conclusion}

We reported on interferometric measurements of Betelgeuse that were obtained with NACO/SAMPol. We detected the polarized signature interpreted as a scattered-light shell located at $1.5 R \star$ using a thin dust shell model. A model that includes a $\sim 4.5$ mas extension of the shell would result in a $10-20 \%$ variation in 
the dust density and grain size. Although the data constrain the shell and size of its constituent grains, they cannot alone disentangle the composition of dust. However, such a study can be attempted using comparison of the dust shell models with SPHERE/ZIMPOL measurements. We tend to favor the ironfree silicates such as forsterite for the dust composition rather than alumina, although no single species was able to perfectly reproduce the data. It is possible that the new dusty structure reported here could form the base of a scattering-driven wind following the theoretical predictions reported in Höfner (2008). In order to proceed in the characterization of the inner dust shell of Betelgeuse, polarimetric observables should be monitored in more spectral bands, particularly in the visible continuum. Close to the photosphere, it is likely that the dust shell is affected by changes in surface luminosity that are due to convection and so variability at timescales of some months is expected.

Acknowledgements. This research has made use of the Jean-Marie Mariotti Center SearchCal service ${ }^{4}$ that is co-developed by FIZEAU and LAOG/IPAG, and of CDS Astronomical Databases SIMBAD and VIZIER ${ }^{5}$. X.H. thanks Cornelis Dullemond for the time he spent supporting preliminary simulations on RADMC3D as well as Susanne Höfner and Bernd Freytag for helpful discussions.

\section{References}

Aurière, M., Donati, J.-F., Konstantinova-Antova, R., et al. 2010, A\&A, 516, L2 Bonneau, D., Clausse, J.-M., Delfosse, X., et al. 2006, A\&A, 456, 789

Castelli, F., \& Kurucz, R. L. 2003, Modelling of Stellar Atmospheres, 210, 20P

Dehaes, S., Bauwens, E., Decin, L., et al. 2011, A\&A, 533, A107

Draine, B. T. 1981, Physical Processes in Red Giants: Proceedings of the Second Workshop, Erice, Italy, September 3-13, 1980 (Dordrecht: D. Reidel Publishing Co.), 88, 317

Ducati, J. R. 2002, VizieR Online Data Catalog: II/237
Harper, G. M., Brown, A., Guinan, E. F., et al. 2017, AJ, 154, 11 Haubois, X., Perrin, G., Lacour, S., et al. 2009, A\&A, 508, 923 Hayes, D. P. 1984, ApJS, 55, 179

Höfner, S. 2008, A\&A, 491, L1

Ireland, M. J., Tuthill, P. G., Davis, J., \& Tango, W. 2005, MNRAS, 361, 337 Jäger, C., Dorschner, J., Mutschke, H., Posch, T., \& Henning, T. 2003, A\&A, 408, 193

Kamiński, T., Gottlieb, C. A., Schmidt, M. R., et al. 2013, EAS Pub. Ser., 60, 191 Karovska, M., Nisenson, P., \& Noyes, R. 1986, ApJ, 308, 260

Kervella, P., Perrin, G., Chiavassa, A., et al. 2011, A\&A, 531, A117

Kervella, P., Le Bertre, T., \& Perrin, G. 2013, EAS Publ. Ser., 60

Kervella, P., Lagadec, E., Montargès, M., et al. 2016, A\&A, 585, A28

Khouri, T., Maercker, M., Waters, L. B. F. M., et al. 2016, A\&A, 591, A70

Kostogryz, N. M., Milic, I., Berdyugina, S. V., \& Hauschildt, P. H. 2016, A\&A, 586, A 87

Le Borgne, J. F., Mauron, N., \& Leroy, J. L. 1986, A\&A, 168, 21

Lenzen, R., Hartung, M., Brandner, W., et al. 2003, Proc. SPIE, 4841, 944

Mauron, N., \& Le Borgne, J. F. 1986, A\&A, 168, 217

Monnier, J. D., Geballe, T. R., \& Danchi, W. C. 1998, ApJ, 502, 833

Montargès, M., Kervella, P., Perrin, G., et al. 2014, A\&A, 572, A17

Montargès, M., Kervella, P., Perrin, G., et al. 2016, A\&A, 588, A130

Norris, B. R. M., Tuthill, P. G., Ireland, M. J., et al. 2012, Nature, 484, 220

O'Gorman, E., Vlemmings, W., Richards, A. M. S., et al. 2015, Revolution in Astronomy with ALMA: The Third Year (San Francisco: ASP), 499, 335

Ohnaka, K., Weigelt, G., \& Hofmann, K.-H. 2016, A\&A, 589, A91

Ohnaka, K., Weigelt, G., \& Hofmann, K.-H. 2017, A\&A, 597, A20

Perrin, G., Ridgway, S. T., Coudé du Foresto, V., et al. 2004, A\&A, 418, 675

Perrin, G., Verhoelst, T., Ridgway, S. T., et al. 2007, A\&A, 474, 599

Pinte, C., Ménard, F., Duchêne, G., \& Bastien, P. 2006, A\&A, 459, 797

Pinte, C., Harries, T. J., Min, M., et al. 2009, A\&A, 498, 967

Ravi, V., Wishnow, E. H., Townes, C. H., et al. 2011, ApJ, 740, 24

Richichi, A., \& Roccatagliata, V. 2005, A\&A, 433, 305

Roddier, F., \& Roddier, C. 1985, ApJ, 295, L21

Rousset, G., Lacombe, F., Puget, P., et al. 2003, Proc. SPIE, 4839, 140

Scicluna, P., Siebenmorgen, R., Wesson, R., et al. 2015, A\&A, 584, L10

Tsuji, T. 1978, PASJ, 30, 435

van Loon, J. T., Marshall, J. R., \& Zijlstra, A. A. 2005, A\&A, 442, 597

Verhoelst, T., Decin, L., van Malderen, R., et al. 2006, A\&A, 447, 311

Woitke, P., Kamp, I., \& Thi, W.-F. 2009, A\&A, 501, 383

\footnotetext{
4 Available at http: //wwW . jmmc . fr/searchcal

5 Available at http://cdsweb.u-strasbg.fr/
} 
Appendix A: Additional figures
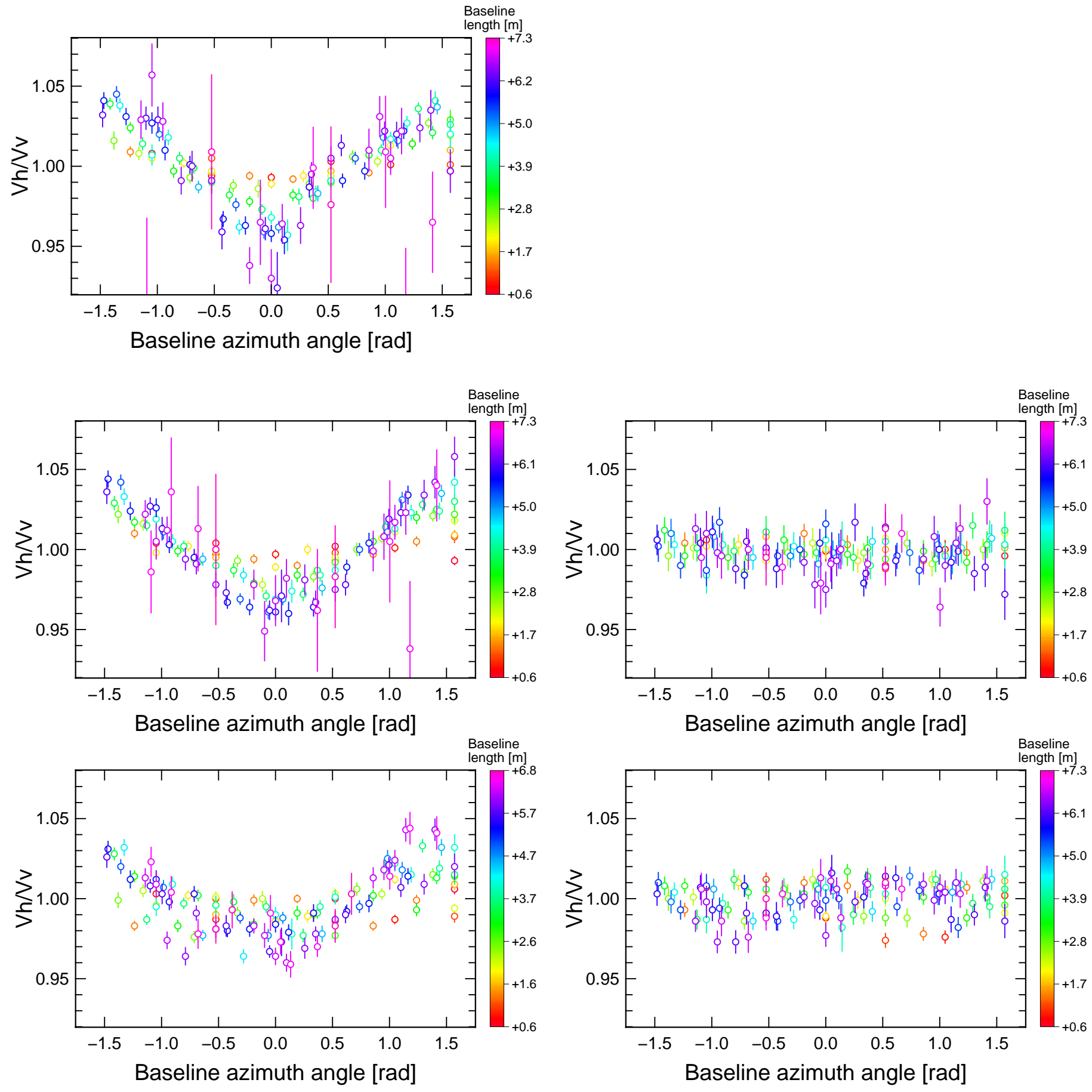

Fig. A.1. Differential visibility ratio plotted as a function of azimuth angle, color-coded with baseline length. Left: Betelgeuse. Right: Aldebaran. From top to bottom: filters centered on 1.08, 1.09, and $1.24 \mu \mathrm{m}$. We did not observe Aldebaran in the 1.08 filter. 
A\&A 628, A101 (2019)

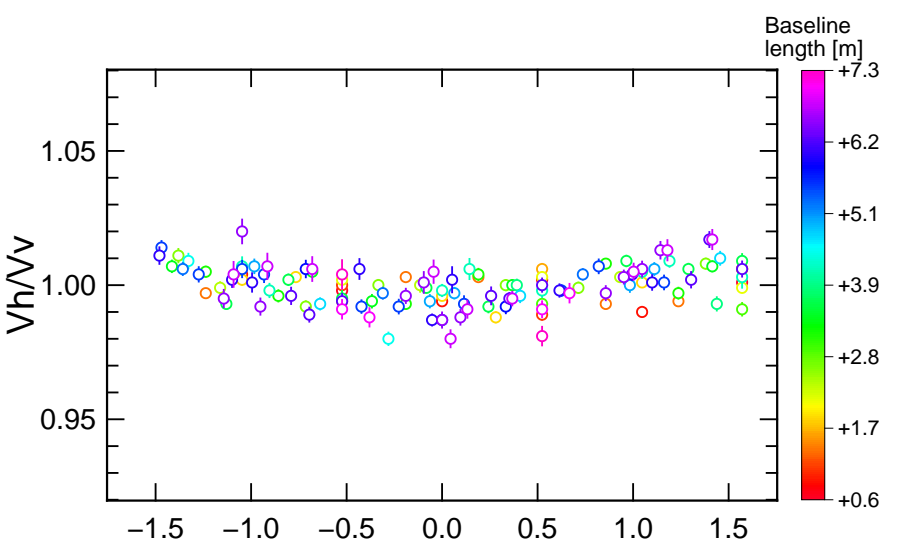

Baseline azimuth angle [rad]

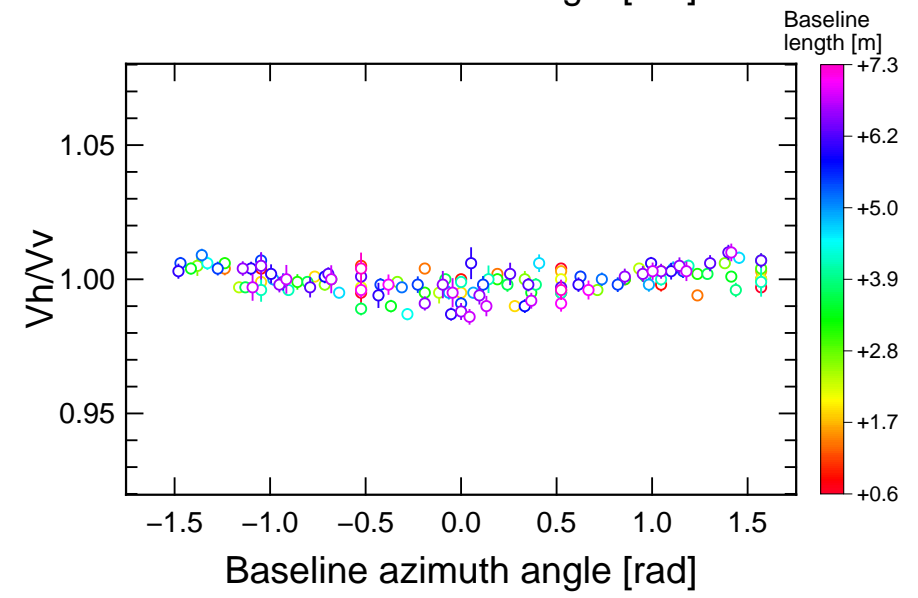

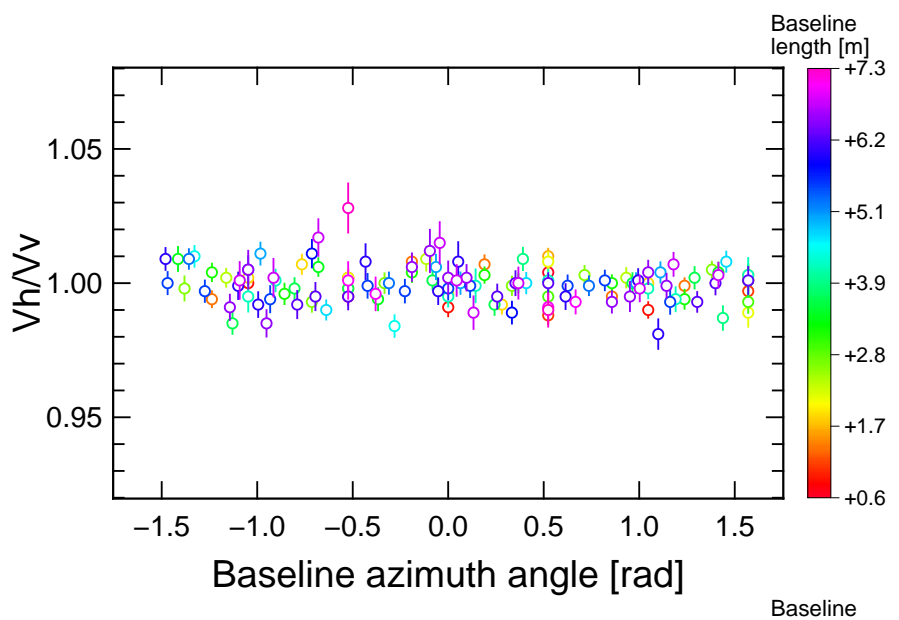

length $[\mathrm{m}]$

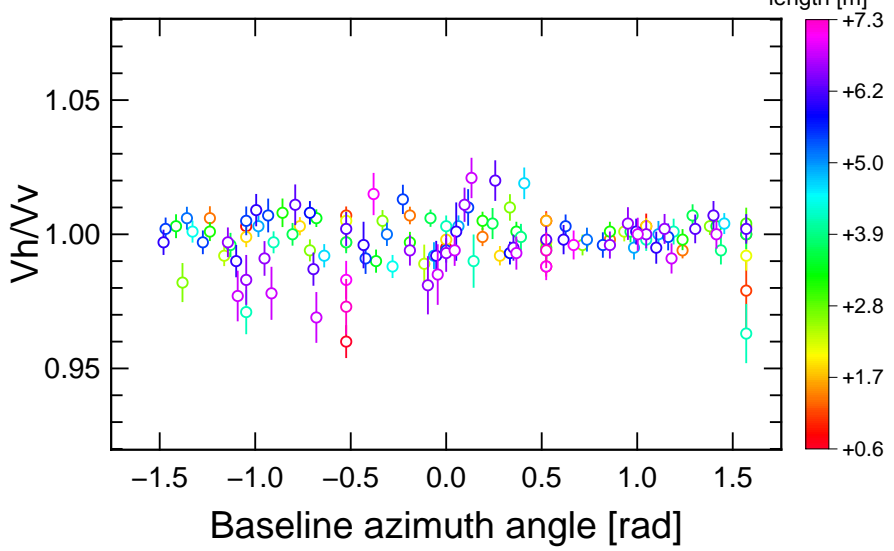

Fig. A.2. Same as Fig. A.1 for filters centered on 1.64 (top) and $1.75 \mu \mathrm{m}$ (bottom). 